\title{
Pleasure, the Common Currency of Emotions
}

\author{
J. MARTIN RAMIREZ ${ }^{a}$ AND MICHEL CABANAC ${ }^{b}$ \\ ${ }^{a}$ Department of Psychobiology and Institute for Biofunctional Studies, \\ Universidad Complutense, Madrid, Spain \\ ${ }^{b}$ Département de Physiologie, Université Laval, Quebec, Canada
}

KEYWORDS: emotion; cognition; pleasure; anger; experience; expression

The old controversy on whether emotion is independent of cognition ${ }^{1,2}$ or dependent on $\mathrm{it}^{3,4}$ has now advanced considerably. The solution fundamentally depends on how one defines both terms, emotion and cognition. Most authors would agree that emotion could be el icited in the absence of conscious cognition mediation. But, if "cognitive" is taken in a broad sense including basic sensory information processing, virtually all agree that some cognitive processing is required for most, if not all, emotions. ${ }^{5}$ And even if emotion - at least in its b roadest meaning, including feelings, mood, and temperament - can be e licited with $m$ inimal $c$ ognitive $p$ rerequisites, $v$ ia noncognitive routes, ${ }^{6}$ the cognitive appraisal is putatively necessary for its elicitation. $^{7}$

Physiologically, there is a two-way interaction between emotion and cognition made possible by the bidirectional connections existent between the main anatomical structures subserving both of them, the amygdala and the neocortex,${ }^{8}$ and by the distribution of neuropeptides in their circuits. ${ }^{9}$

As a follow-up to a definition of sensation as a four-dimensional experience (quality, intensity, hedonicity, and duration), ${ }^{10}$ and accepting that sensation is the origin of all consciousness, that model should apply to all forms of mental experience, ${ }^{11}$ including emotion, which is just a special case of consciousness: if emotion takes place in co nsciousness, it s hould also possess those four dimensions. Ac tually, $\mathrm{C}$ abanac ${ }^{12}$ proposes that emotion is any mental experience with high intensity and high hedonic content, expanding on a thesis that motivational states can be compared to each other by a common currency, which would be pleasure. 
This conception, which facilitates the understanding of emotions, can be reached by introspective intuition, but direct evidence of its validity can also be tested by deduction, based on experimental results, such as those obtained by our research group, which described verbal analysis of the mental experience and the expression of anger in different cultures ${ }^{13,14}$ and the relationship between aggression, impulsiveness, and hedonicity. ${ }^{15}$ We recognize that all these elements possess a strongly hedonic dimension, either positive or negative.

Generalizing our findings to other emotions, it may be concluded that hedonicity would be the dimension of consciousness that motivates the subject towards useful behaviors. This contention matches with Damasio's ${ }^{16,17}$ observation that impairment of emotional process in patients undermines their capacity to make decisions: this is what one would expect to find when the hedonic dimension is severed. The lack of pleasure thus impairs emotion.

Consequently, pleasure/displeasure is the common currency for accessing behavior in response to the various emotions; no emotion is hedonically indifferent. The he donic dimension is what pathogno monically defines emotion. ${ }^{12,18}$ Pleasure thus makes emotion a motivating experience.

\section{ACKNOWLEDGMENTS}

This work was supported by the Spanish Ministry of Science and Technology (BS2001/1224) and by the Spanish Interministerial Commission for Science and Technology ( CICYT) (PR 111/01).

\section{REFERENCES}

1. CAnnon, W.B. 1927. The James-Lange theory of emotions: a critical examination and an alternation. Am. J. Psychol. 39: 106-124.

2. LAZARUS, R.S. 1982. Tho ughts on the relations between e motion and cognition. Am. Psychol. 37: 1019-1024.

3. James, W. 1890. The Principles of Psychology. Dover Publications. New York.

4. Zajonc, R.B. 1984. On the primacy of affect. Am. Psychol. 39: 117-123.

5. DAvidson, R.J. \& P. EKMAN. 1994. Afterword: what are the minimal cognitive prerequisites for emotion? In The Nature of Emotion. P. Ekman \& R.J. Davidson, Eds.: 232-234. Oxford University Press. New York.

6. IZARD, C.E. 1994. Cognition is one of four types of emotion activating systems. In The Nature of Emotion. P. Ekman \& R.J. Davidson, Eds.: 230-207. Oxford University Press. New York.

7. Fridja, N.H. 1994. Emotions require cognitions, even if simple ones. In The Nature of Em otion. P. Ekman \& R.J. Davidson, Eds.: 197-202. Oxford University Press. New York. 
8. LeDoux, J. 19 96. The E motional Br ain. T he M ysterious U nderpinnings of Emotional Life. Simon \& Schuster. New York.

9. PAKSEPP, J. 1986. The a natomy of emotions. In Emotion: Theory, Res earch, and Experience. R. Plutchik \& H. Kellerman, Eds.: 91-124. Academic Press. Orlando, FL.

10. Cabanac, M. 1979. Sensory pleasure. Quarterly Rev. Biol. 54: 1-29.

11. Cabanac, M. 1996. On the origin of consciousness, a postulate and its corollary. Neurosci. Biobehav. Rev. 20: 33-40.

12. Cabanac, M. 2002, What is emotion? Behav. Processes 60: $69-83$.

13. Ramirez, J.M., T. Fujihara, S. van Goozen \& C. Santisteban. 2001. Anger proneness in Japanese and Spanish students. In Cross-cultural Approaches to Aggression and Reconciliation. J. Martin Ramirez \& Deborah R. Richardson, Eds.: 87-97. NovaScience. Huntington, NY.

14. Ramirez, J.M., C. Santisteban, T. Fujihara \& S. van Goozen. 2002. Differences between experience of anger and readiness to angry action (a study of Japanese and Spanish students). Aggressive Behav. 28: 429-438.

15. Ramirez, J.M., M.C. Bonniot-CabanaC \& M. CabanaC. Impulsive aggression can provide pleasure. A s tudy with people of different ages. Submitted for publication.

16. Damasio, A.R. 1994. Descartes' Error. Putnam. New York.

17. Damasio, A.R. 2003. Looking for Spinoza: Joy, Sorrow and the Human Brain. Harcourt Trade Publishers. New York.

18. Rolls, E.T. 1994. A theory of emotion and consciousness, and its application to understanding the ne ural ba sis of e motion. In $\mathrm{T}$ he $\mathrm{C}$ ognitive Neurosciences. M.S. Gazzaniga, Ed.: 1091-1105. MIT Press. Boston.

published? 